\title{
A THEOREM ON AN ANALYTIC MAPPING OF RIEMANN SURFACES
}

\author{
MINORU KURITA
}

Recently S. S. Chern [1] intended an aproach to some problems about analytic mappings of Riemann surfaces from a view-point of differential geometry. In that line we treat here orders of circular points of analytic mappings. The author expresses his thanks to Prof. K. Noshiro for his kind advices.

1. In the first we summarise formulas for conformal mappings of Riemannian manifolds (cf. [3], [4]). We take 2-dimensional Riemannian manifolds $M$ and $N$ of differentiable class $C_{2}$ and assume that there exists a conformal mapping $f$ of $M$ into $N$ which is locally diffeomorphic. We take orthonormal frames on the tangent spaces of points of any neighborhood $U$ of $M$. Then a line element $d s$ of $M$ can be represented as

$$
d s^{2}=\omega_{1}^{2}+\omega_{2}^{2}
$$

with 1-forms $\omega_{1}$ and $\omega_{2}$. When we choose frames on the tangent spaces of $f(U)$ corresponding to those on $M$ by the conformal mapping $f$, we have for a line element $d t$ of $N$

$$
d t^{2}=\pi_{1}^{2}+\pi_{2}^{2}=a^{2} d s^{2}
$$

where

$$
\pi_{1}=a \omega_{1}, \quad \pi_{2}=a \omega_{2} . \quad(a>0)
$$

A form $\omega_{12}$ of Riemannian connection of $M$ is defined in $U$ by the relations

$$
d \omega_{1}=\omega_{2} \wedge \omega_{21}, \quad d \omega_{2}=\omega_{1} \wedge \omega_{12}, \quad \omega_{12}=-\omega_{21} .
$$

When we put

$$
d a / a=b_{1} \omega_{1}+b_{2} \omega_{2},
$$

a connection form $\pi_{12}=-\pi_{21}$ of Riemannian connection of $N$ in $f(U)$ is given by

$$
\pi_{12}=\omega_{12}+b_{1} \omega_{2}-b_{2} \omega_{1} .
$$

Received April 28, 1961. 
In fact we have then by virtue of $(3),(4),(5),(6)$

$$
d \pi_{1}=\pi_{2} \wedge \pi_{21}, \quad d \pi_{2}=\pi_{1} \wedge \pi_{12} .
$$

Curvature forms of $M$ and $N$ are given by

$$
\Omega_{12}=-d \omega_{12}=k d S, \quad \Pi_{12}=-d \pi_{12}=h d T,
$$

where $k, h$ are Gaussian curvatures and $d S, d T$ are surface elements of $M$ and $N$ respectively. By taking exterior differentials of (6) we get

$$
\Pi_{12}=\Omega_{12}-d\left(b_{1} \omega_{2}-b_{2} \omega_{1}\right) .
$$

Next we put

$$
b^{2}=b_{1}^{2}+b_{2}^{2}
$$

and

$$
\begin{aligned}
& d b_{1}+b_{2} \omega_{21}-b_{1} d a / a+\frac{1}{2} b^{2} \omega_{1}=\lambda_{11} \omega_{1}+\lambda_{12} \omega_{2} \\
& d b_{2}+b_{1} \omega_{12}-b_{2} d a / a+\frac{1}{2} b^{2} \omega_{2}=\lambda_{21} \omega_{1}+\lambda_{22} \omega_{2} .
\end{aligned}
$$

Calculating $d(d a / a)=d\left(b_{1} \omega_{1}+b_{2} \omega_{2}\right)=0$ we have $\lambda_{12}=\lambda_{21}$, and $\left(\lambda_{i j}\right)$ are components of a symmetric tensor on $M$.

On the Riemannian manifold $M$ covariant differential $\left(D l_{1}, D l_{2}\right)$ of a vector field with orthogonal components $\left(l_{1}, l_{2}\right)$ is given by $D l_{1}=d l_{1}+l_{2} \omega_{21}, D l_{2}$ $=d l_{2}+l_{1} \omega_{12}$, and

$$
\tau=\frac{l_{1} D l_{2}-l_{2} D l_{1}}{l_{1}^{2}+l_{2}^{2}}=\frac{l_{1} d l_{2}-l_{2} d l_{1}}{l_{1}^{2}+l_{2}^{2}}+\omega_{12}=d\left(\tan ^{-1} \frac{l_{2}}{l_{1}}\right)+\omega_{12}
$$

gives a form on $M . \theta=\tan ^{-1}\left(l_{2} / l_{1}\right)$ is an angle between the vector $\left(l_{1}, l_{2}\right)$ and the first axis of the orthonormal frame (cf. [5]). We consider eigendirections of an arbitrary symmetric tensor field $\left(\lambda_{i j}\right)$. At a point where two eigenvalues are different an eigendirection corresponding to a greater one is determined. An angle $\theta$ between the eigendirection and the first axis is given by $\tan 2 \theta=\frac{2 \lambda_{12}}{\lambda_{11}-\lambda_{22}}$ and (11) reduces to

$$
\tau=\frac{1}{2} d\left(\tan ^{-1} \frac{2 \lambda_{12}}{\lambda_{11}-\lambda_{22}}\right)+\omega_{12}
$$

Next we take complex frames such that

$$
\rho=\omega_{1}+\sqrt{-1} \omega_{2}, \quad \bar{\rho}=\omega_{1}-\sqrt{-1} \omega_{2} .
$$

Then (1) and (4) reduce to 


$$
d s^{2}=\rho \bar{\rho}, \quad d \rho=\sqrt{ }-1 \rho \wedge \alpha,
$$

where we have put $\omega_{12}=\alpha$. Putting

$$
d a / a=b_{1} \omega_{1}+b_{2} \omega_{2}=c \rho+\bar{c} \bar{\rho}
$$

we get

$$
b_{1}=c+\bar{c}, \quad b_{2}=\sqrt{-1} \overline{1}(c-\bar{c})
$$

and $(8)$ reduces to

$$
\Pi_{12}=\Omega_{12}+\sqrt{-1} d(c \rho-\bar{c} \bar{\rho}) .
$$

For a symmetric tensor $\left(\lambda_{i j}\right)$ we put

$$
Q=\lambda_{11} \omega_{1}^{2}+2 \lambda_{12} \omega_{1} \omega_{2}+\lambda_{22} \omega_{2}^{2}=L \rho^{2}+2 M \rho \bar{\rho}+N \bar{\rho}^{2}
$$

and we get

$$
\lambda_{11}=L+\bar{L}+2 M, \quad \lambda_{12}=\sqrt{-1}(L-\bar{L}), \quad \lambda_{22}=-L-\bar{L}+2 M .
$$

Hence (12) reduces to

$$
\tau=\frac{\sqrt{-1}}{4}\left(\frac{d L}{\bar{L}}-\frac{d \bar{L}}{\bar{L}}\right)+\alpha
$$

For $\boldsymbol{Q}=\sum_{i j} \lambda_{i j} \omega_{i} \omega_{j}$ corresponding to $\left(\lambda_{i j}\right)$ defined by (10) we have

$$
Q=\left(d b_{1}+b_{2} \omega_{21}\right) \omega_{1}+\left(d b_{2}+b_{1} \omega_{12}\right) \omega_{2}-(d a / a)^{2}+\frac{1}{2} b^{2}\left(\omega_{1}^{2}+\omega_{2}^{2}\right) .
$$

Taking (16) and $\omega_{1}=\frac{1}{2}(\rho+\bar{\rho}), \omega_{2}=\frac{-\sqrt{ }-1}{2}(\rho-\bar{\rho})$ into consideration we get

$$
Q=(d c-\sqrt{ }-1 \alpha c) \rho+(d \bar{c}+\sqrt{-1} \alpha \bar{c}) \bar{\rho}-c^{2} \rho^{2}-\bar{c}^{2} \bar{\rho}^{2} .
$$

When we put

$$
d c-\sqrt{ }-1 \alpha c=l \rho+m \bar{\rho},
$$

we have

$$
d \bar{c}+\sqrt{ }-1 \alpha \bar{c}=\bar{m} \rho+\bar{l} \bar{\rho} .
$$

By the relation $d(d a / a)=0$ we get $m=\bar{m}$.

$L$ and $M$ corresponding to $\left(\lambda_{i j}\right)$ defined by (10) are

$$
L=l-c^{2}, \quad M=m .
$$

by virtue of (18), (20), (21).

2. Now we take up Riemann surfaces (complex manifolds of dimension 1) $M$ and $N$ and an analytic mapping $f$ of a domain $D(\subset M)$ into $N . \quad M$ and $N$ can be endowed with Riemannian metrics of constant Gaussian curvature $k$ 
and $h$ respectively, and the line elements $d s$ and $d t$ can be represented in suitably chosen coordinates $z$ and $w$ as

with

$$
\begin{array}{cl}
d s^{2}=p^{2} d z d \bar{z}, & d t^{2}=q^{2} d w d \bar{w} \\
p=(1+k / 4 z \bar{z})^{-1}, & q=(1+h / 4 w \bar{w})^{-1} .
\end{array}
$$

Signs of $k$ and $h$ coinside with those of Euler's characteristics of $M$ and $N$. The analytic mapping $f$ is represented in our coordinates as $w=f(z)$ with an analytic function $f(z)$. This analytic mapping $f$ induces conformal mapping in the neighborhood of any point such that $f^{\prime}(z) \neq 0$ and we have $d t^{2}=a^{2} d s^{2}$ with

$$
a^{2}=\left(q^{2} / p^{2}\right) f^{\prime} \bar{f}^{\prime}
$$

We put

$$
\rho=p d z
$$

then we have

$$
d s^{2}=\rho \bar{\rho}
$$

and a real 1-form $\alpha$ satisfying (14) is given uniquely by

$$
\alpha=\sqrt{-1}\left(-\frac{\partial \log p}{\partial z} d z+\frac{\partial \log p}{\partial \bar{z}} d \bar{z}\right) .
$$

Putting

$$
f^{\prime \prime} / f^{\prime}=\varphi
$$

we get from (25)

$$
d a / a=d(\log q)-d(\log p)+\frac{1}{2}(\varphi d z+\bar{\varphi} d \bar{z}) .
$$

Hence by the relations (15), (26) we have

$$
p c=\frac{\partial \log q}{\partial w} f^{\prime}-\frac{\partial \log p}{\partial z}+\frac{1}{2} \varphi .
$$

By virtue of (24) $\frac{\partial \log p}{\partial z}=-\frac{k}{4} \bar{z} p, \quad \frac{\partial \log q}{\partial w}=-\frac{h}{4} \bar{w} q$,

and so

$$
p c=-\frac{h}{4} \bar{w} q f^{\prime}+\frac{k}{4} \bar{z} p+\frac{1}{2} \varphi .
$$

Next we have

$$
p(d c-\sqrt{-1} \alpha c)=d(p c)-c d p-\sqrt{-1} \alpha p c .
$$

This is equal to $p(l \rho+m \bar{\rho})=p^{2}(l d z+m d \bar{z})$ by (21), and we get by (31), (26) (28), (30) 


$$
\begin{aligned}
p^{2} l & =-\frac{h}{4} \bar{w} \frac{\partial q}{\partial w}\left(f^{\prime}\right)^{2}-\frac{h}{4} \bar{w} q f^{\prime \prime}+\frac{k}{4} z \frac{\partial p}{\partial z}+\frac{1}{2} \varphi^{\prime}-2 c \frac{\partial p}{\partial z} \\
& =\left(\frac{h}{4} \bar{w} q f^{\prime}\right)^{2}-\frac{h}{4} \bar{w} q f^{\prime \prime}-\left(\frac{k}{4} \bar{z} p\right)^{2}+\frac{1}{2} \varphi^{\prime}+2 p c \frac{k}{4} \bar{z} p,
\end{aligned}
$$

and so

$$
p^{2} l-p^{2} c^{2}=\left(\frac{h}{4} \bar{w} q f^{\prime}\right)^{2}-\frac{h}{4} \bar{w} q f^{\prime \prime}+\frac{1}{2} \varphi^{\prime}-\left(\frac{k}{4} \bar{z} p-p c\right)^{2} .
$$

By the relation $(31)$

Putting

$$
\frac{k}{4} \bar{z} p-p c=\frac{h}{4} \bar{w} q f^{\prime}-\frac{1}{2} \varphi
$$

and we get

$$
p^{2}\left(l-c^{2}\right)=\frac{1}{2}\left(\varphi^{\prime}-\frac{1}{2} \varphi^{2}\right)
$$

$$
\emptyset=\varphi^{\prime}-\frac{1}{2} \cdot \varphi^{2}
$$

we have

$$
L=l-c^{2}=\frac{1}{2 p^{2}} \Phi
$$

Thus for eigendirections of $\left(\lambda_{i j}\right)$ defined by (10) $L$ in (19) is this one.

3. Now we integrate (17). We take a bounded domain $D(\subset M)$ and assume that an analytic function $f(z)$ induced from an analytic mapping $f$ of $D$ into $N$ in any coordinate neighborhood has not a singular point. A property that $f(z)$ is stationary (i.e. $f^{\prime}(z)=0$ ) at a point is independent of complex local coordinates. We take a point $p_{i}(i=1, \ldots, s)$ which is a stationary point and a small simply connected domain $D_{i}$ about $p_{i}$ with a boundary curve $c_{i}$. Orientations of the boundary curve $c$ of $D$ and $c_{i}$ are determined by that of D. Now

$$
\nu=b_{1} \omega_{2}-b_{2} \omega_{1}=-\sqrt{-1}(c \rho-\bar{c} \bar{\rho})
$$

is a form on $D$. We integrate (17) and get by Stokes' theorem

$$
\int_{D^{\prime}} f^{*}\left(\Pi_{12}\right)=\int_{D^{\prime}} \Omega_{12}-\int_{c} \nu+\sum_{i} \int_{c_{i}} \nu
$$

where $f^{*}$ denotes a mapping dual to $f$ of forms on $N$ to those on $M$ and $D^{\prime}=D-\sum_{i} D_{i} . \quad$ By the relation (31)

$$
\begin{aligned}
\nu & =-\sqrt{ }-1(p c d z-p \bar{c} d \bar{z}) \\
& =\sqrt{-1}\left(\frac{h}{4} q(\bar{w} d w-w d \bar{w})-\frac{k}{4} p(\bar{z} d z-z d \bar{z})+\frac{1}{2}(\varphi d z-\bar{\varphi} d \bar{z})\right) .
\end{aligned}
$$

We take local coordinates $z$ and $w$ for which (24) holds good and $z=0$ and 
$w=f(0)=0$ at a point. This is possible because the metric (23) is homogeneous. Thus when a point $p_{i}$ with a local coordinates $z=0$ is a stationary point of an analytic function $f(z)$, we have

$$
f(z)=a z^{m}+b z^{m+1}+\cdots . \quad(a \neq 0, m>1)
$$

Thus

$$
\varphi=\frac{f^{\prime \prime}}{f^{\prime}}=\frac{1}{z}\left((m-1)+\frac{m+1}{m} \cdot \frac{b}{a} z+\cdots\right)
$$

Contracting $c_{i}$ to the point $p_{i}$ we get by (36)

$$
\lim \int_{c_{i}} \nu=2 \pi(m-1),
$$

because integrations of the first and the second terms in (36) tend to 0 as $z$ does so. We denote $m_{i}$ anew instead of $m$, and we get

$$
\int_{D} f^{*}(h d T)=\int_{D} k d S-\int_{C} \nu+2 \pi \sum_{i}\left(m_{i}-1\right) .
$$

If $D$ is compact and $n$-leaved covering surface of $f(D)$, we have

$$
n \chi(f(D))=\chi(D)+\sum_{i}\left(m_{i}-1\right)
$$

where $\chi$ means Euler's characteristic. This is Hurwitz's formula. Our derivation of this formula is closely related to that of Chern's (cf. [1]). A difference lies in the point that $\nu$ in (38) which is defined by (35) has an intrinsic meaning on $M$ and $d_{\nu}=\Delta(\log a) d S$, where $\Delta$ means a Laplacian operator.

4. Next we integrate (19) for $L$ given by (34). We take a domain $D$ and assume that it contains stationary points $p_{i}(l=1, \ldots, s)$ and points $q_{j}(j=1$, $\ldots, r)$ at which $\emptyset(z)=0$. We call these $q_{j}$ circular points of our mapping. This definition is independent of local coordinates. $\Phi(z)=0$ is equivalent to an ambiguity of eigendirections of the tensor $\left(\lambda_{i j}\right)$. We take small simply connected domains $D_{i}$ and $E_{j}$ containing the points $p_{i}$ and $q_{j}$ and bounded by curves $c_{i}$ and $e_{j}$ respectively. Then by orientations of the boundary $c$ of $D$ and $c_{i}, e_{j}$ corresponding to an orientation of $D$ we get from (19)

$$
\int_{c} \tau-\sum_{i} \int_{c_{i}} \tau-\sum_{j} \int_{e_{j}} \tau=\int_{D^{\prime}} d \tau=-\int_{D^{\prime}} k d S,
$$

where $D^{\prime}=D-\sum D_{i}-\sum E_{j}$. We take local coordinates $z$ in $M$ and $w$ in $N$ in such a way that $z=0$ at $p_{i}$ and $f(0)=0$. We have by virtue of (19) and (34) 


$$
\int_{c_{i}} \tau=\frac{\sqrt{-1}}{4} \int_{c_{i}}\left(\frac{d \Phi}{\Phi}-\frac{d \bar{\Phi}}{\bar{\Phi}}\right)+\int_{c_{i}} \alpha
$$

When we contract $c_{i}$ to the point $p_{i}$, the second term on the right side vanishes, because $\int_{C_{i}} \alpha=\int_{D_{i}} d \alpha=-\int_{D_{i}} k d S$, and we get

$$
\lim \int_{c_{i}} \tau=\lim \frac{\sqrt{-1}}{4} \int_{c_{i}}\left(\frac{d \Phi}{\Phi}-\frac{d \bar{\Phi}}{\emptyset}\right)
$$

Around a stationary point we have

$$
f(z)=a z^{n}+b z^{n+1}+\cdots . \quad(a \neq 0, n>1)
$$

Then

$$
\begin{gathered}
\varphi=\frac{f^{\prime \prime}}{f^{\prime}}=\frac{1}{z}\left(n-1+\frac{n+1}{n} \frac{b}{a} z+\cdots\right) \\
\emptyset=\varphi^{\prime}-\frac{1}{2} \varphi^{2}=-\frac{n^{2}-1}{2 z^{2}} g(z)+\cdots,
\end{gathered}
$$

where

$$
g(z)=1+\frac{2}{n} \frac{b}{a} z+\cdots .
$$

Hence

$$
\frac{d \Phi}{\Phi}=\left(-\frac{2}{z}+\frac{g^{\prime}}{g}\right) d z
$$

and so

$$
\lim \int_{c_{i}} \tau=2 \pi \text {. }
$$

Next we consider circular points $q_{j}$. Taking a local coordinate $z$ such that $z=0$ at $q_{j}$ we can put

$$
\Phi=a z^{n}+b z^{n+1}+\cdots \quad(a \neq 0)
$$

We call $n$ an order of the circular point $q_{j}$. This is independent of local coordinates, as is seen below in (42). We have in this case

$$
\frac{d \emptyset}{\emptyset}=\left(\frac{n}{z}+\cdots\right) d z
$$

and when we contract $e_{j}$ to $q_{j}$, we get $\lim \int_{e_{j}} \frac{d \Phi}{\Phi}=2 n \pi \sqrt{-1}$

and so

$$
\lim \int_{e_{j}} \tau=-n \pi
$$

We denote $n$ at $q_{j}$ as $n_{j}$ anew and we get by (40), (41), (42)

$$
\int_{c} \tau-2 \pi s+\pi \sum_{j} n_{j}=\int_{D} d \alpha=-\int_{D} k d S
$$


If $M$ is compact and $f$ is an analytic mapping of $M$ into $N$, we get for Euler's characteristic $\%(M)$

$$
\%(M)=s-\frac{1}{2} \sum_{j} n_{j}
$$

by virtue of Gauss-Bonnet's formula $2 \pi \chi(M)=\int_{M} k d S$. Thus we have got a final result.

TheOREM. We assume that $M$ and $N$ are closed Riemann surfaces endowed with Hermitian metrics of constant curvature, and $f$ is an analytic mapping of $M$ onto $N$, of which the number of stationary points is $s$ and the orders of circular points are $n_{j}(j=1, \ldots, r)$. Then we have the relation (44).

Finally we give an interpretation of the relation $\Phi=0$, which means a vanishing of a Schwarzian derivative of $f(z)$. We consider a circular point and take a local coordinate $z$ in $M$ and $w$ in $N$ for which (24) holds good and $z=0$ at the point and also $w=f(0)=0$. Then we can put

$$
f(z)=a z+b z^{2}+c z^{3}+\cdots . \quad(a \neq 0)
$$

Hence $\quad \varphi=\frac{f^{\prime \prime}}{f^{\prime}}=(2 b+6 c z+\cdots) \cdot \frac{1}{a}\left(1+\frac{2 b}{a} z+\cdots\right)^{-1}$

$$
=\frac{2 b}{a}+\left(\frac{6 c}{a}-\frac{4 b^{2}}{a^{2}}\right) z+\cdots
$$

and so

$$
\left.\emptyset=\frac{6}{a}\left(c-\frac{b^{2}}{a}\right)+0, z\right) .
$$

$\Phi(0)=0$ means $c=b^{2} / a$ and then

$$
f(z)=a z\left(1+\frac{b}{a} z+\left(\frac{b}{a} z\right)^{2}+\cdots\right)=\frac{a^{2} z}{a-b z}+0\left(z^{4}\right) .
$$

Thus $f(z)$ is a linear fractional function with disregard to terms of order greater than 3 .

We have named a circular point the point at which $\Phi=0$, namely an eigendirection of the mapping is ambiguous. About twenty years ago A. Fialkow and K. Yano studied a conformal transformation for which $\left(\lambda_{i j}\right)$ is a multiple of a unit matrix and Yano called it concircular because it transforms a Riemannian circle into a same. A circular point is a one at which $f$ is nearly concircular. 


\section{REFERENCES}

[1] Chern, S. S.; Complex analytic mappings on Riemann surfaces. Amer. Jour. Math. vol. 82 (1960), pp. 323-337.

[2] Fialkow, A.; Conformal geodesics, Trans. Amer. Math. Soc. vol. 45 (1939), pp. 443-473.

[3] Kurita, M.; On conformal Riemann spaces. Jour. Math. Soc. Japan vol. 7 (1955), pp. $13-31$.

[4] Kurita, M.; On the holonomy group of the conformally flat Riemannian manifold, Nagoya Math. Jour. vol. 9 (1955), pp. 161-171.

[5] Kurita, M.; A note on umbilics of a closed surface. Nagoya Math. Jour. vol. 15 (1959), pp.

[6] Yano, K.; Concircular geometry, I. Concircular transformations. Proc. Imp. Acad. Japan (1940) pp. 195-200.

Mathematical Institute

Nagoya University 